\title{
The impact of the COVID-19 outbreak on emergency surgery: a Greek emergency department experience
}

\author{
Francesk Mulita, Michail Vailas, Levan Tchabashvili, Elias Liolis, Fotios Iliopoulos, Nikolas Drakos, \\ Ioannis Maroulis \\ Department of Surgery, General University Hospital of Patras, Patras, Greece
}

Gastroenterology Rev 2021; 16 (1): 95

DOI: https://doi.org/10.5114/pg.2021.104739

\begin{abstract}
Address for correspondence: Dr. Francesk Mulita, Department of Surgery, General University Hospital of Patras, Greece,
\end{abstract} e-mail: oknarfmulita@hotmail.com

The coronavirus disease (COVID-19) outbreak caused by severe acute respiratory syndrome coronavirus 2 (SARS-CoV-2) has become a global health problem. COVID-19 is a highly infectious and multifaceted disease, and already millions of people have been infected worldwide [1]. According to the literature, the COVID-19 pandemic caused a major reduction of emergency surgical operations as well as overall admissions to emergency departments because of the widespread hospital fear and anxiety experienced by most patients during the peak of this outbreak [2].

We report herein our experience from a tertiary General University Hospital in Greece, covering a population of approximately 1.5 million people. There is no doubt that the causes of the sharp reduction of emergency surgical cases during the COVID-19 pandemic are multi-factorial, reflecting a dangerous situation with serious consequences for public health.

In our correspondence, we report the number of operations we have performed, comparing periods before and after the start of the COVID-19 pandemic (Table I). According to the results of our study, we did not observe a statistically significant difference between the total numbers of patients admitted for acute conditions be- tween the 2 examined periods. However, our data show that hospital stay, as well as the operation duration of patients in the COVID era, has increased, which is a fact that reflects the severity of the clinical presentation of these patients. Patients in the COVID era presented to the hospital with delayed onset of symptoms, in comparison with those admitted before the COVID pandemic, due to their anxiety and fear of being infected with the coronavirus.

\section{Conflict of interest}

The authors declare no conflict of interest.

\section{References}

1. Saberian P, Mireskandari SM, Baratloo A, et al. antibody Rapid Test Results in Emergency Medical Services Personnel during COVID-19 pandemic: a cross sectional study. Arch Acad Emerg Med 2020; 9: e2.

2. Castagneto-Gissey L, Casella G, Russo MF, et al. Impact of COVID-19 outbreak on emergency surgery and emergency department admissions: an Italian level 2 emergency department experience. Br J Surg 2020; 107: e374-5.

Received: 20.01.2021

Accepted: 15.03 .2021

Table I. Emergency surgeries before and after the COVID-19 pandemic outbreak (26/2/2019-26/12/2019 vs. 26/02/2020-26/12/2020)

\begin{tabular}{lccc} 
Variable & $\begin{array}{c}\text { Pre-COVID-19 } \\
\text { 26.02.2019-26.12.2019 }\end{array}$ & $\begin{array}{c}\text { COVID-19 era } \\
\mathbf{2 6 . 0 2 . 2 0 2 0 - 2 6 . 1 2 . 2 0 2 0}\end{array}$ & $\begin{array}{c}\text { Statistical significance } \\
\boldsymbol{p} \mathbf{0 . 0 5}\end{array}$ \\
\hline Number of patients $(n=965)$ & 494 & 471 & NS \\
\hline Males/females (479/486) & $252 / 242$ & $227 / 244$ & NS \\
\hline Mean age [years] & 48.6 & 47.9 & NS \\
\hline Mean hospitalization [days] & 4.17 & 4.49 & $<0.05$ \\
\hline Mean operative \pm SD time $[\mathrm{min}]$ & 103.1 & 109.7 & $<.05$
\end{tabular}

NS - not significant. 